\title{
Gene Therapy for Primary Immunodeficiencies
}

\author{
Christine Rivat,, ${ }^{1,2}$ Giorgia Santilli, H. Bobby Gaspar,, ${ }^{1,2}$ and Adrian J. Thrasher ${ }^{1,2}$
}

\begin{abstract}
For over 40 years, primary immunodeficiencies (PIDs) have featured prominently in the development and refinement of human allogeneic hematopoietic stem cell transplantation. More recently, ex vivo somatic gene therapy using autologous cells has provided remarkable evidence of clinical efficacy in patients without HLAmatched stem cell donors and in whom toxicity of allogeneic procedures is likely to be high. Together with improved preclinical models, a wealth of information has accumulated that has allowed development of safer, more sophisticated technologies and protocols that are applicable to a much broader range of diseases. In this review we summarize the status of these gene therapy trials and discuss the emerging application of similar strategies to other PIDs.
\end{abstract}

\section{Primary Immunodeficiencies as Targets for Gene Therapy}

$\mathbf{P}$ Rimary immunodeficiencies (PIDs) are a diverse group of over 300 genetic disorders that fundamentally affect the development and/or functionality of the immune system. Most of them are rare monogenic disorders, but the spectrum of PIDs is constantly expanding with the identification of novel immunodeficiency syndromes through next generation sequencing technologies and improved clinical awareness. Patients classically present with a higher susceptibility to infections or infection with unusual organisms and may also develop autoimmunity or autoinflammatory disease and lymphoreticular malignancies. Although minimal or supportive therapies are effective for many of these conditions, the severest require definitive early treatment in order to prevent chronic morbidity and early mortality. Allogeneic hematopoietic stem cell (HSC) transplantation has in particular proved to be a highly effective curative option for many PIDs caused by intrinsic hematopoietic gene defects. Although associated with morbidity and mortality directly related to conditioning chemotherapy and graft-versus-host disease, newer protocols using reduced intensity regimens and alternative sources of stem cells such as umbilical cord blood (which allows more flexibility in terms of HLA matching) are proving to be increasingly safe and efficacious. Even so, there are large numbers of patients for whom HLAmatched donors are unavailable and for whom an autologous procedure based on genetic correction of hematopoietic stem and progenitor cells is a highly attractive option. Surprisingly, gene therapy may also prove to be even more economically viable because the associated healthcare costs compared with conventional therapies are likely to be significantly lower. On the other hand, this provides a difficult business model for commercialization of gene therapies, particularly since severe PIDs are individually quite rare. Considerable advances have been made in gene transfer technology over the last 20 years with the development of refined retroviral vector technologies that may allow both safer and more effective gene transfer and expression. Stem cell culture conditions and transduction protocols have also been improved to enhance cell viability and gene transfer efficiency during ex vivo procedures (Cooray et al., 2012).

\section{Updates on Clinical Trials for Individual PIDs}

\section{$A D A-S C I D$}

The first primary immune deficiency to be treated with gene therapy was the adenosine deaminase-severe combined immuno deficiency (ADA-SCID). In the absence of adenosine deaminase (ADA), the accumulation of toxic purine metabolites in the thymus has deleterious effects on lymphocyte development and function. ADA-deficient patients have a characteristic immunological profile with TB-NK- SCID, resulting in recurrent infections and failure to thrive, but because ADA is ubiquitously expressed, ADAdeficient patients also show skeletal, lung, liver, gastrointestinal tract, and neural abnormalities. Although exogenous polyethylene glycol-conjugated ADA (PEG-ADA) therapy can successfully rescue the immune system of ADA-deficient patients and reduce the incidence of infections, these effects are often at best partial and poorly sustained (Chan et al., 2005). The first attempts at HSC gammaretroviral gene

${ }^{1} \mathrm{UCL}$ Institute of Child Health, Centre for Immunodeficiency, London WCIN 1EH, United Kingdom.

${ }^{2}$ Great Ormond Street Hospital for Children, National Health Service Trust, London WCIN 1EH, United Kingdom. 
therapy for this disease produced only low levels of genemarked cells in peripheral blood, with no obvious clinical benefit to the patients who therefore remained on enzyme replacement therapy (Aiuti et al., 2002; Bordignon et al., 1995). This has been attributed to suboptimal cell culture and transduction conditions at the time and to the continuous use of PEG-ADA, which may have compromised the expected survival and developmental advantage that introduction of the $A D A$ gene should confer to lymphocyte precursors and their progeny. However, failure to engraft sufficient numbers of progenitor cells appeared to be a more fundamental problem. In a new generation of trials, PEG-ADA was discontinued prior to gene therapy in order to restore the selective advantage of gene-corrected cells, and probably more importantly, patients received low-intensity alkylating agent conditioning to promote the long-term engraftment of transplanted HSCs and progenitors in the bone marrow. Several phase I/II trials using variations of this modified protocol and long terminal repeat (LTR)-regulated gammaretroviral vectors were conducted in Italy, London, and the United States, including to date a total of over 38 patients (Table 1). All patients are alive, and over $70 \%$ have shown sufficient levels of immune reconstitution and metabolic detoxification to justify the cessation of PEG-ADA. After a median follow-up of 4 years in 10 patients treated in Italy, the majority of patients had high levels of gene marking in $\mathrm{T}$, B, and NK cells (88\%, 52\%, and 59\%, respectively) and persistent transgene expression in granulocytes, monocytes, and megakaryocytes $(0.1 \%-10 \%)$, demonstrating the successful engraftment of gene-marked long-lived progenitors and potentially multipotent HSCs. These patients had evidence of substantial immune reconstitution, with increased lymphocyte counts and a polyclonal $\mathrm{T}$-cell repertoire, associated with evidence of restored thymic activity and systemic metabolic detoxification. In five patients, immunoglobulin replacement therapy was discontinued, and good humoral response to vaccination was observed (Aiuti et al., 2009). In the United Kingdom, four of eight patients treated with a similar protocol and a spleen focus-forming virus (SFFV) LTR-based gammaretroviral vector also showed high levels of gene marking and sustained immunological and metabolic correction of the disease (Gaspar et al., 2011b). Interestingly, one patient in London who demonstrated only partial immune recovery after gene therapy improved considerably in terms of T-lymphocyte gene marking after reinitiation of PEG-ADA, suggesting that in some situations the administration of PEG-ADA can also promote the expansion of gene-modified cells.

No serious events have been encountered so far following gene therapy for ADA-SCID. In five patients treated with gene therapy, integration sites were analyzed before infusion of transduced CD34 HSC and progenitor cells and in bone marrow and peripheral cells up to 4 years after treatment. Integration hotspots were identified in transcriptionally active regions, including sites near the proto-oncogenes $L M O-2, B C L 2$, and CCND2, but to date there has been no evidence of clonal dysregulation (Aiuti et al., 2007). Despite the unquestionable clinical benefit of gene therapy for ADA patients, 30\% have had to recommence PEG-ADA support. The relative failure of gene therapy in these cases can probably be attributed either to poor stem cell harvest doses and the infusion of low numbers of transduced cells or to an insufficient recovery of thymic function. It is also interesting to note that in patients treated with an SFFV LTR-based vector in London, there is some molecular evidence for overrepresentation of insertion sites near the Evi1 locus and also for a degree of LTR promoter methylation suggestive of expression silencing (Thrasher, unpublished results).

\section{$X$-linked SCID}

X-linked SCID (SCID-X1) is the most common form of severe combined immunodeficiency, accounting for $40 \%-50 \%$ of all cases. Mutations in the IL2RG gene lead to defective expression of the common gamma chain $(\gamma \mathrm{c})$, a subunit shared by multiple cytokine receptors, including the interleukin (IL)-2, IL-4, IL-7, IL-9, IL-15, and IL-21 receptor complexes, which are involved in the development and function of all lymphocytes. As a consequence, patients show profound cellular and humoral defects, resulting from the low number or absence of $\mathrm{T}$ and natural killer (NK) lymphocytes, and the loss of function of B lymphocytes (T-B + NK - SCID) (Noguchi et al., 1993). Two clinical trials, including a total number of 20 subjects were conducted in France and the United Kingdom using virtually identical LTR-regulated gammaretroviral vectors and very similar transduction protocols (Table 1) (Gaspar et al., 2011a; HaceinBey-Abina et al., 2010). In both studies, transduced HSCs were re-infused to the patients without preconditioning, with the expectation that the extremely high survival advantage of transduced progenitor cells would allow the expansion of a functional gene-corrected population. Overall, the outcome of these trials was very positive, with high gene marking in T cells, associated with minimal levels in other lineages, reflecting a low level of engraftment of multipotent HSC, as expected from what was an unconditioned procedure (i.e., there was no selective pressure for HSC engraftment, either intrinsic to the disease or created by chemotherapeutic ablation). Two older patients treated did not reconstitute, likely as a result of an age-dependent restriction on thymic recovery (Thrasher et al., 2005). The rapid and sustained increase in T-cell numbers, and to a lesser extent in NK cells, was associated with a normal phenotypic $\mathrm{T}$ cell receptor (TCR) repertoire, proliferative potential, and capacity to clear common viral infections. The restoration of humoral immunity was variable, but approximately one half of patients were able to discontinue immunoglobulin replacement therapy. Regrettably (and surprisingly at the time), 2 to 6 years after treatment five patients who were clinically well developed T-cell acute lymphoblastic leukemia (T-ALL) resulting from mutagenic retroviral insertions (Hacein-Bey-Abina et al., 2008; Howe et al., 2008). In four out of five patients enhancer-mediated up-regulation of the LMO-2 proto-oncogene was directly implicated in the leukemic process, although an accumulation of more classical genetic changes unrelated to retroviral vector insertion were probably required for final evolution to acute T-ALL. All patients were treated with relatively standard chemotherapy, but one patient died of refractory leukemia. The other four are now in long-term remission with sustained partial restoration of immunity. A third trial using a similar protocol and vector was conducted in the United States, with the difference that the patients enrolled were preadolescent 


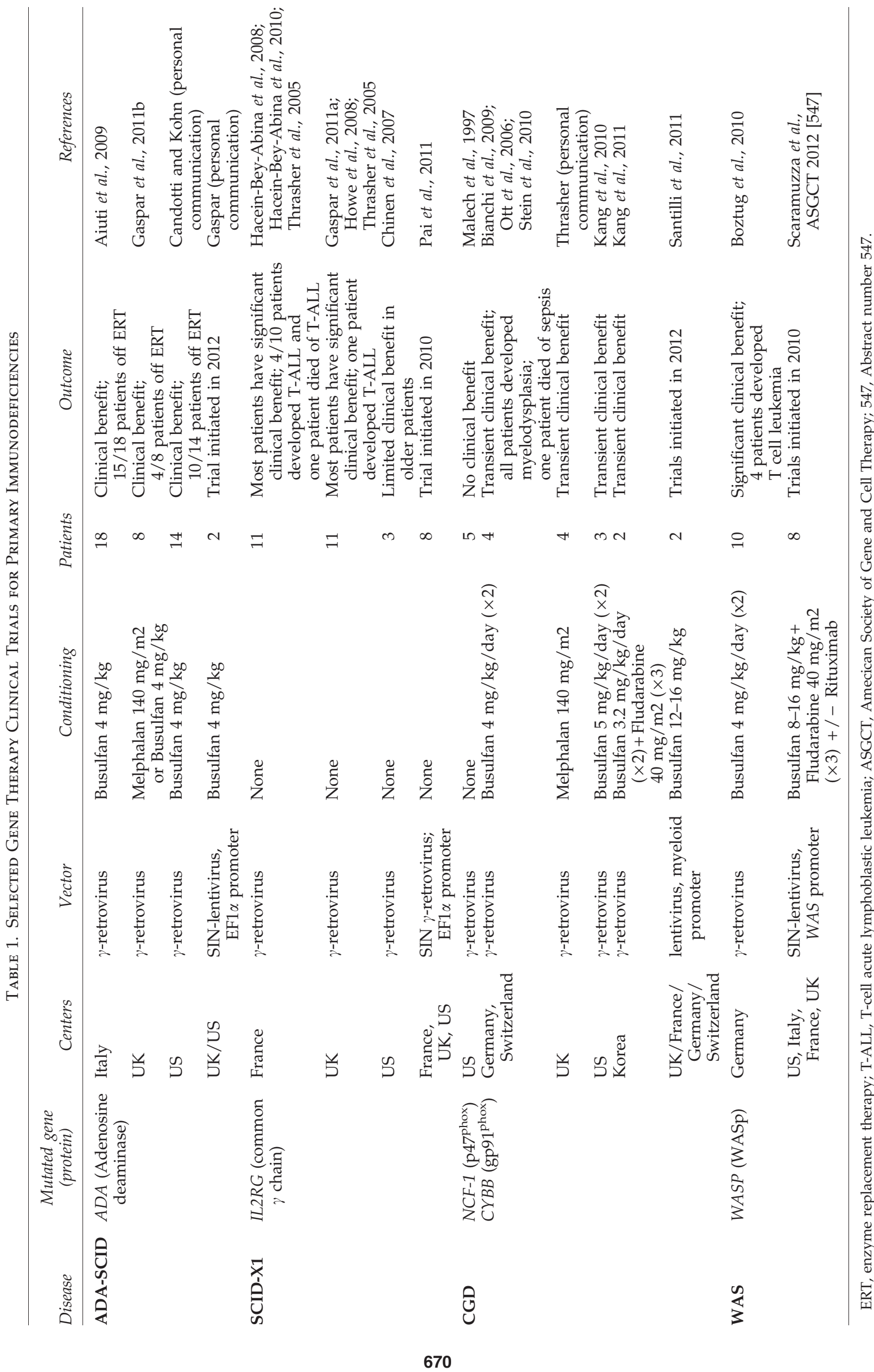


subjects aged 10 to 14 years, who had previously been treated unsuccessfully with bone marrow transplant. Immune reconstitution was limited in these patients despite good engraftment, with only a slight improvement of T-cell function in the youngest child. This unexpectedly reduced efficacy of gene therapy is likely explained by the age-related decreased plasticity and loss of thymopoietic capacity in older subjects particularly those in whom thymopoiesis has been absent for extended periods (Chinen et al., 2007).

\section{Chronic granulomatous disease}

Chronic granulomatous disease (CGD) is caused by defects in the nicotinamide adenine dinucleotide phosphate oxidase (NADPH-oxidase), which catalyses the oxidative burst during phagocytosis, resulting in impaired antimicrobial killing activity by neutrophils and recurrent life-threatening bacterial and fungal infections. In the majority of cases, CGD is due to mutations in the CYBB gene, which encodes the gp91 ${ }^{\text {phox }}$ component of the NADPHoxidase complex, although a significant proportion have mutations in NCF2, which encodes $\mathrm{p} 47^{\text {phox }}$. The first HSC gene therapy trial for this disease was initiated in the United States without chemotherapy, but only achieved short-term engraftment with no long-lasting benefit for patients, outlining the likely necessity to condition patients before treatment since CGD-corrected cells are not predicted to have any selective growth or survival advantage (Malech et al., 1997). More recently, five early phase clinical studies involving a total of 12 patients and incorporating low-intensity alkylating agent conditioning (Table 1) (Grez et al., 2011) have been conducted in several centers worldwide. Overall, these demonstrated biologically and clinically significant restoration of NADPH-oxidase activity in circulating neutrophils for a short time after engraftment, allowing the majority of patients to clear pre-existing infections. However, clinical benefit was usually only transient, and gene marking rapidly decreased, with only a few patients having significant marking after 3 months, indicating an absence of long-term engraftment despite the use of low-intensity preconditioning (Bianchi et al., 2009; Kang et al., 2010, 2011; Ott et al., 2006). Once again, several patients treated with an SFFV LTR-based gammaretroviral vector developed an unexpected increase in the number of functionally corrected neutrophils over time as a result of insertional transactivation of myeloproliferative genes. Eventually, however, gene expression was silenced in the majority of these cells through CpG dinucleotide methylation at the retroviral promoter, even though enhancer activity and therefore mutagenic potential was preserved (Stein et al., 2010).

\section{Wiskott-Aldrich syndrome}

Wiskott-Aldrich syndrome (WAS) is another rare X-linked immunodeficiency, in which deficiency of WAS protein (WASp), which is critically involved in the cytoskeletal dynamics of hematopoietic cells, leads to varied cellular immunological dysfunctions and severe platelet defects (microthrombocytopenia). Data from a recent trial in Germany using highly efficient conventional gammaretroviral gene transfer to autologous cells and low-intensity conditioning have been reported (Table 1). Over 2 years after gene therapy, sustained WASp expression was detected in all hematopoietic lineages with up to $90 \%$ gene marking in T cells (in part due to selective accumulation of corrected cells), but long-term engraftment of HSC and progenitors was suggested by the detection of $10 \%-20 \%$ marked bone marrow $\mathrm{CD}^{+} 4^{+}$cells. These observations were associated with an increase in platelet counts and correction of leukocyte functions, including NK cell-mediated cytotoxicity, monocyte migration, immunoglobulin production, and T-lymphocyte proliferation. Clinically, patients were less susceptible to infections, with resolution of eczema and autoimmune disorders (Boztug et al., 2010). However, although detailed mapping of viral integration sites originally showed a polyclonal integration pattern, several patients have developed clonal abnormalities including T-cell leukemia associated with insertions in the vicinity of LMO-2 and other proto-oncogenes (Avedillo Diez et al., 2011). These findings indicate that LMO-2-mediated mutagenesis is a potential outcome arising from use of probably all LTR-based gammaretroviral vectors and that SCID-X1 patients may not necessarily be at higher risk than others. Furthermore, they highlight the fact that frank leukemic transformation is an extremely acute process in general and that clonal tracking may be of little practical utility for predicting occurrence.

\section{Preclinical development of gene therapy for other candidate PIDs}

The success of gene therapy for SCID-X1, ADA-SCID, CGD, and WAS suggests that similar therapeutic strategies could be applied to other primary immune deficiencies (Table 2). JAK3

Table 2. Preclinical Development of Gene Therapy for Other Primary Immunodeficiencies

\begin{tabular}{|c|c|c|c|}
\hline Disease & Mutated gene (protein) & Vector & References \\
\hline Rag1/2 deficiency & RAG1/2 (RAG1/2) & lentivirus/retrovirus & $\begin{array}{l}\text { Pike-Overzet et al., 2011; } \\
\quad \text { Yates et al., } 2002\end{array}$ \\
\hline Artemis deficiency (RS-SCID) & DCLRE1C (Artemis) & lentivirus & Benjelloun et al., 2008 \\
\hline Jak3 deficiency & JAK3 (JAK3) & $\gamma$-retrovirus & McCauslin et al., 2003 \\
\hline X-linked hyper IgM & TNFSF5 (CD40L) & $\gamma$-retrovirus & Brown et al., 1998 \\
\hline X-linked agammaglobulinemia (XLA) & BTK (BTK) & $\gamma$-retrovirus/lentivirus & Yu et al., 2004; Kerns et al., 2010 \\
\hline Leukocyte adhesion defect (LAD)-1 & ITGB2 (CD18) & lentivirus/foamy virus & Hunter et al., 2011 \\
\hline $\begin{array}{l}\text { X-linked lymphoproliferative } \\
\text { syndrome (XLP)-1 }\end{array}$ & SH2D1A (SAP) & lentivirus & $\begin{array}{l}\text { Gaspar (personal } \\
\text { communication) }\end{array}$ \\
\hline $\begin{array}{l}\text { Familial hemophagocytic } \\
\text { lymphohistiocytosis (FHLH) }\end{array}$ & PRF1 (perforin) & lentivirus & $\begin{array}{l}\text { Gaspar (personal } \\
\text { communication) }\end{array}$ \\
\hline
\end{tabular}


is a tyrosine kinase required for signal transduction by the common cytokine receptor gamma chain, and therefore autosomal recessive JAK3 deficiency leads to a phenotype that is identical to SCID-X1, with an absence of circulating T and NK cells, and a partial loss of intrinsic B-cell function. Similarly, IL-7 receptor alpha deficiency results in the same T-cell phenotype, but normal B and NK cells. However, these disorders are extremely rare, making clinical development somewhat unattractive at least at the current time. Other more tractable forms of SCID that are currently under preclinical development include defects in the recombination activating genes RAG1 and RAG2 and in the DCLRE1C gene encoding Artemis. RAG1/2 and Artemis proteins participate in V(D)J recombination, which is essential for the generation of functional B- and $\mathrm{T}$-cell receptors and normal $\mathrm{T}$ - and B-cell development. In all cases, the transfer of genetically modified HSCs can effectively reconstitute murine models of the disease, although correction of Rag1 deficiency required high transgene copy number even with potent LTR enhancer elements, which potentially increases the risk of insertional mutagenesis (Benjelloun et al., 2008; McCauslin et al., 2003; Pike-Overzet et al., 2011; Yates et al., 2002). Elements that may improve the reliability of transgene expression, including the ubiquitous chromatin opening element (UCOE) have recently shown promise in these disorders and may also be useful for other conditions in which safe high-level or tissue-regulated gene expression is desirable at low transgene load (Pike-Overzet et al., 2011; Van Til et al.; Zhang et al., 2010).

X-linked agammaglobulinemia (XLA) is caused by mutations in the Bruton's tyrosine kinase (BTK) gene, an enzyme of the TEC family of kinases expressed during B-cell development and involved in pro-B/pre-B cell transition. The absence of BTK results in an accumulation of pro-B cells and a decrease in numbers of mature $B$ cells and very low or absent serum immunoglobulins. Preclinical murine reconstitution experiments using gammaretrovirus-based gene transfer showed rescue of B-cell differentiation and functions associated with an increase in IgM and IgG3 levels and restored response to T-independent challenge (Yu et al., 2004). Similar results were obtained using a lentiviral vector containing the immunoglobulin enhancer $(\mathrm{Emu})$ and Igbeta (B29) minimal promoter to regulate lineage-specific Btk expression (Kerns et al., 2010). These encouraging results support the development of gene therapy for human XLA. Although there is an existing excellent treatment in the form of immunoglobulin replacement therapy for patients in developed countries, for many populations worldwide (such as in China) this is not always the case, and the argument for development of a single curative gene therapy becomes quite compelling.

X-linked hyper IgM syndrome (X-HIGM1) is another combined immunodeficiency characterized by defects in isotype switching from $\operatorname{IgM}$ to $\operatorname{IgG}$, $\operatorname{Ig} \mathrm{A}$, and $\operatorname{IgE}$, as well as impaired lymphocyte and myeloid functions. X-HIGM1 is associated with mutations in the TNFSF5 gene encoding CD40 ligand (CD40L), a member of the TNF superfamily, which is primarily expressed on activated $\mathrm{T}$ cells and binds the CD40 receptor on the surface of B cells and antigenpresenting cells to regulate B-cell function and inflammatory responses. The animal model for X-HIGM1 is the CD40L knock-out mouse, which recapitulates some of the humoral defects seen in the patients. Retroviral gene transfer suc- cessfully corrected the phenotype of CD40L ${ }^{-/-}$mice, but 12 out of 19 treated animals developed T-lymphoproliferative disorders apparently unrelated to insertional mutagenesis (Brown et al., 1998). These results are in agreement with another study reporting atypical lymphoid proliferation in transgenic mice overexpressing CD40L (Sacco et al., 2000), and highlight the necessity of tightly regulating CD40L expression in order to avoid unexpected detrimental effects. Attempts to correct a murine model by trans-splicing repair were partially effective, although this strategy has not been pursued (Tahara et al., 2004). Leukocyte adhesion deficiency (LAD) type I, which is caused by mutations in the ITGB2 gene encoding the CD18 integrin subunit, is primarily a neutrophil disorder characterized by recurrent life-threatening bacterial infections and poor wound healing. Interestingly, experience from allogeneic transplant indicates that quite low numbers of normal donor neutrophils are sufficient to achieve disease control. However, a preliminary study, in which two patients were treated with gammaretroviral gene therapy in the absence of conditioning, achieved very low $(<0.1 \%)$ levels of gene marking in neutrophils, with no corrected cells detectable in the periphery after 2 months (Bauer and Hickstein, 2000). More recently, the canine model (CLAD) of the disease was successfully treated using HSCs transduced with either lentiviral or foamy viral vectors expressing CD18, which represents an important step towards the development of gene therapy for LAD patients (Hunter et al., 2011).

While most conditions discussed so far are largely dependent on stable gene transfer to long-lasting HSCs, other conditions may also be amenable to therapy by targeting mature T-cell populations. X-linked lymphoproliferative disease (XLP), a T and NK lymphoproliferative disorder due to mutations in the signaling adaptor SLAM-associated protein (SAP), and familial hemophagocytic lymphohistiocytosis (FHLH), a hemophagocytic disease resulting from defective $\mathrm{NK}$ and $\mathrm{CD}^{+}$T-cell cytotoxicity, are potential candidates for both approaches. SAP is involved in multiple signaling pathways affecting proliferation, apoptosis, and differentiation, while perforin, which is the most commonly mutated protein in FHLH, is a direct effector of cytotoxicity. Lineage-restricted expression of these genes might be required to avoid deleterious effects in nontarget cells. In these conditions, preclinical efficacy and safety studies using improved viral vectors are currently being undertaken in order to determine clinical applicability.

\section{Ongoing Challenges to Safety and Efficacy}

\section{Clonal dominance and malignant expansion}

One known feature of gammaretroviruses is their tendency to integrate into sites of active chromatin, resulting in a higher probability of insertion near regulatory regions such as transcription start sites of genes that are highly expressed in early hematopoietic precursors. The recent analysis of the distribution of insertion sites in the engrafted cells of HSC gene therapy patients indicates a reproducible integration profile, with over $40 \%$ of the viral insertions occurring in genomic areas that only represent $0.36 \%$ of the genome (Cattoglio et al., 2010; Deichmann et al., 2011). In addition, the mapping of the insertion sites in the SCID-X1, CGD, and WAS gene therapy patients with evidence of benign or malignant clonal expansion revealed integration hotspots in the 
vicinity of a limited number of genes. In SCID-X1 and WAS patients, the development of T-ALL was linked to insertion of the vector in T cells near the proto-oncogene LIM domain only-2 (LMO-2) and the cell cycle regulators BMI1 and CCND2, all of which have leukemogenic properties. Insertion into these sites led to aberrant and nonphysiological transcription of the proto-oncogenes through transactivation from the enhancer elements of the viral LTR. Other genetic and chromosomal abnormalities were also detected in the blast populations of these patients, including gain of function NOTCH1 mutations and deletion of the tumor suppressor gene CDKN2A (cyclin-dependent kinase 2A). Interestingly, ADA patients showed a similar overall integration profile, with insertions near LMO-2, CCND2, BCL2, DYRK1A, and $B L M$, but no evidence to date of clonal selection. This suggests that ADA deficiency is the exception, and that there may be intrinsic metabolic or proliferative defects that restrict leukemogenic potential in this disease even in the presence of detrimental insertions. In CGD patients, integration site analyses revealed preferential insertion near the proto-oncogenes PRDM16, MDS1/EVI1, SETBP1, or CCDN2, associated in most cases with myeloproliferation, suggesting that the disease background plays a significant role in the phenotype of emerging clonal dominance.

\section{Current Clinical Activities and the Future for PID Gene Therapy}

\section{Vector developments}

Considerable effort has gone into development and testing of vectors that are less likely to cause significant insertional mutagenesis and toxicity, for example, through elimination of endogenous retroviral enhancer sequences. Self-inactivating (SIN) gammaretroviral vectors have recently entered clinical study as part of a trans-Atlantic collaboration and are showing early promise in patients with SCID-X1 treated in the United Kingdom, the United States, and France (Pai et al., 2011). The multicenter model of testing the same vector in a rare disease such as SCID-X1 has proved to be particularly effective for sharing of resources and will enable expedited recruitment of informative patient numbers and early determination of efficacy and safety. This will greatly facilitate the adoption of protocol and technological refinements and perhaps eventually product registration. SIN lentiviral vectors have also been developed for SCID-X1, one of which has been successfully incorporated into a clinical grade stable producer cell line by concatemeric transfection, thereby greatly facilitating the production process (Throm et al., 2009). Similarly, lentiviral vectors using housekeeping promoter elements for ADA deficiency or natural regulatory sequences for WAS have recently entered the clinical arena in multicenter efforts (Boztug et al., 2006; Charrier et al., 2005; Montiel-Equihua et al., 2012) and are being developed for other PIDs as detailed previously. Once again, early reports are of encouraging efficacy with no toxicity, although followup time is so far relatively short. Regulated or physiological gene expression is desirable not only for safety (because it will restrict potential deleterious enhancer activity), but may also enhance efficacy. Various tissue specific elements have been incorporated into viral vectors. Among those are the CD19 promoter for expression in B cells (Moreau et al., 2004), the CD4 gene promoter/enhancer sequences (Marodon et al.,
2003) or the Lck promoter for targeted expression in T cells (Lois et al., 2002), and the 3.2-kb dectin-2 gene fragment to restrict the expression to antigen-presenting cells (Bonkobara et al., 2001). More recently the $5^{\prime}$ flanking regions of C-Fes and cathepsin $\mathrm{G}$ myeloid genes have been fused to create a chimeric element that efficiently achieves biochemical correction of X-CGD neutrophils both in vitro and in vivo (Santilli et al., 2011). First into man efforts with this vector in Europe using the "specials" exemption to advanced therapy medicinal product (ATMP) legislation have provided useful information and demonstrated good activity in humans (Thrasher, unpublished data). Similarly, myeloid specific promoters derived from $C D 11 B$ and $C D 18$ (IGTB2) genes have been used to correct CLAD (Hunter et al., 2011). Following on from LTR-based studies in Germany, a WAS gene minimal promoter was experimentally selected to regulate WASp expression in a lentiviral vector for trials now open in Italy, France, the United Kingdom, and the United States (Charrier et al., 2007; Scaramuzza et al., 2012). An alternative approach for selective expression is detargeting through the use of microRNA (miR) recognition sequences. For instance miR-126 and miR-130a are expressed in HSCs and early progenitors from both mice and humans, but not in differentiated cells. By incorporating miR-126 target sequences into therapeutic vectors, it is possible to limit expression in HSCs while maintaining robust expression in mature hematopoietic cells. This approach has recently been used for the gene therapy of a globoid cell leukodystrophy mouse model with promising results (Gentner et al., 2010) and is under investigation for certain PIDs (for example, FHLH and CGD) in which gene expression in early HSCs and progenitors may be undesirable.

\section{Gene therapy by homologous recombination}

An alternative approach to gene therapy is to correct the disease-causing mutations in situ by replacing the mutated gene with a functional copy at its own locus. This would allow physiological regulation of gene transcription and protein expression. A gene editing strategy uses the capacity of the cell machinery to repair double-stranded breaks in the DNA by homologous recombination and requires an exogenous DNA template that contains the corrected genomic sequence. Site-specific gene targeting can be achieved using artificial endonucleases, such as homing endonucleases, zinc-finger nucleases, and most recently transcription activatorlike effector nucleases (TALENs). Recently, homing endonucleases specific for the human RAG1 locus have been designed and successfully used for HR-mediated DNA repair in cells (Munoz et al., 2011). Zinc-finger nucleases are another type of engineered endonucleases, in which custommade zinc-finger DNA-binding domains are fused to a dimer of the Fok1 restriction enzyme nuclease domain to introduce double-stranded breaks in chosen target DNA sequences. This approach has been used to correct common gamma chain $(\gamma \mathrm{c})$ mutations in cell lines with relatively good efficiency, suggesting that a similar strategy could be applicable in vivo particularly because of the potent selective advantage imparted to corrected cells (Lombardo et al., 2007). Although genome editing with custom-designed nucleases allows the targeted delivery of therapeutic genes in vitro, the requirement for multiple components to be transferred makes the 
technique complicated to translate clinically and at present the process is inefficient in primary hematopoietic cells. In addition, there is a risk of cellular toxicity due to off-target cleavage, meaning that considerable work on relevant preclinical models is required before clinical consideration.

\section{Conclusions}

PIDs have played a significant part in the development of allogeneic HSC transplantation, and over the last decade have shown that somatic gene therapy is a viable and effective approach in patients. Probably around 100 patients with various forms of PID have been treated worldwide with remarkably little toxicity, even taking into account known cases of insertional mutagenesis. At the same time, the evidence for clinical benefit in several diseases is compelling. Many challenges need to be overcome before gene therapy can be offered as a routine treatment and before a much wider range of diseases become successful targets. These challenges lie not only in the design of safer vectors or in the incorporation of expression elements to achieve clinical efficacy and in the optimization of cell culture methods to preserve viability and function, but also in preparative conditioning of patients to ensure long-term engraftment of HSC or more mature cells using standard chemotherapeutic regimens and potentially serotherapy (Grez et al., 2011). At the same time, allogeneic HSC transplantation has become much more sophisticated and flexible through use of alternative stem cell sources and less toxic conditioning regimens. It seems likely that both strategies will continue to develop alongside each other, which can only be good news for patients with these often devastating diseases.

\section{Acknowledgments}

The authors acknowledge the support of the Wellcome Trust (AJT), the GOSH Children's Charity (AJT and HBG), the Medical Research council (AJT), and the European Commission FP7 CELL-PID (AJT and GS).

\section{Author Disclosure Statement}

No competing financial interests exist.

\section{References}

Aiuti, A., Slavin, S., Aker, M., et al. (2002). Correction of ADASCID by stem cell gene therapy combined with nonmyeloablative conditioning. Science 296, 2410-2413.

Aiuti, A., Cassani, B., Andolfi, G., et al. (2007). Multilineage hematopoietic reconstitution without clonal selection in ADASCID patients treated with stem cell gene therapy. J. Clin. Invest. 117, 2233-2240.

Aiuti, A., Cattaneo, F., Galimberti, S., et al. (2009). Gene therapy for immunodeficiency due to adenosine deaminase deficiency. N. Engl. J. Med. 360, 447-458.

Avedillo Diez, I., Zychlinski, D., Coci, E.G., et al. (2011). Development of novel efficient SIN vectors with improved safety features for Wiskott-Aldrich syndrome stem cell based gene therapy. Mol. Pharm. 8, 1525-1537.

Bauer, T.R., Jr., Hickstein, D.D., (2000). Gene therapy for leukocyte adhesion deficiency. Curr. Opin. Mol. Ther. 2, 383-388.

Benjelloun, F., Garrigue, A., Demerens-de Chappedelaine, C., et al. (2008). Stable and functional lymphoid reconstitution in arte- mis-deficient mice following lentiviral artemis gene transfer into hematopoietic stem cells. Mol. Ther. 16, 1490-1499.

Bianchi, M., Hakkim, A., Brinkmann, V., et al. (2009). Restoration of NET formation by gene therapy in CGD controls aspergillosis. Blood 114, 2619-2622.

Bonkobara, M., Zukas, P.K., Shikano, S., Nakamura, S., et al. (2001). Epidermal Langerhans cell-targeted gene expression by a dectin-2 promoter. J. Immunol. 167, 6893-6900.

Bordignon, C., Notarangelo, L.D., Nobili, N., et al. (1995). Gene therapy in peripheral blood lymphocytes and bone marrow for ADA- immunodeficient patients. Science 270, 470-475.

Boztug, K., Dewey, R.A., and Klein, C. (2006). Development of hematopoietic stem cell gene therapy for Wiskott-Aldrich syndrome. Curr. Opin. Mol. Ther. 8, 390-395.

Boztug, K., Schmidt, M., Schwarzer, A., et al. (2010). Stem-cell gene therapy for the Wiskott-Aldrich syndrome. N. Engl. J. Med. 363, 1918-1927.

Brown, M.P., Topham, D.J., Sangster, M.Y., et al. (1998). Thymic lymphoproliferative disease after successful correction of CD40 ligand deficiency by gene transfer in mice. Nat. Med. 4, 1253-1260.

Cattoglio, C., Pellin, D., Rizzi, E., et al. (2010). High-definition mapping of retroviral integration sites identifies active regulatory elements in human multipotent hematopoietic progenitors. Blood 116, 5507-5517.

Chan, B., Wara, D., Bastian, J., et al. (2005). Long-term efficacy of enzyme replacement therapy for adenosine deaminase (ADA)deficient severe combined immunodeficiency (SCID). Clin. Immunol. 117, 133-143.

Charrier, S., Stockholm, D., Seye, K., et al. (2005). A lentiviral vector encoding the human Wiskott-Aldrich syndrome protein corrects immune and cytoskeletal defects in WASP knockout mice. Gene Ther. 12, 597-606.

Charrier, S., Dupre, L., Scaramuzza, S., et al. (2007). Lentiviral vectors targeting WASp expression to hematopoietic cells, efficiently transduce and correct cells from WAS patients. Gene Ther. 14, 415-428.

Chinen, J., Davis, J., De Ravin, S.S., et al. (2007). Gene therapy improves immune function in preadolescents with $\mathrm{X}$-linked severe combined immunodeficiency. Blood 110, 67-73.

Cooray, S., Howe, S.J., and Thrasher, A.J. (2012). Retrovirus and lentivirus vector design and methods of cell conditioning. Methods Enzymol. 507, 29-57.

Deichmann, A., Brugman, M.H., Bartholomae, C.C., et al. (2011). Insertion sites in engrafted cells cluster within a limited repertoire of genomic areas after gammaretroviral vector gene therapy. Mol. Ther. 19, 2031-2039.

Gaspar, H.B., Cooray, S., Gilmour, K.C., et al. 2011a. Long-term persistence of a polyclonal $\mathrm{T}$ cell repertoire after gene therapy for X-linked severe combined immunodeficiency. Sci. Transl. Med. 3, 97ra79.

Gaspar, H.B., Cooray, S., Gilmour, K.C., et al. 2011b. Hematopoietic stem cell gene therapy for adenosine deaminase-deficient severe combined immunodeficiency leads to long-term immunological recovery and metabolic correction. Sci. Transl. Med. 3, 97ra80.

Gentner, B., Visigalli, I., Hiramatsu, H., et al. (2010). Identification of hematopoietic stem cell-specific miRNAs enables gene therapy of globoid cell leukodystrophy. Sci. Transl. Med. 2, 58ra84. Grez, M., Reichenbach, J., Schwable, J., et al., (2011). Gene therapy of chronic granulomatous disease: the engraftment dilemma. Mol. Ther. 19, 28-35.

Hacein-Bey-Abina, S., Garrigue, A., Wang, G.P., et al. (2008). Insertional oncogenesis in 4 patients after retrovirus-mediated gene therapy of SCID-X1. J. Clin. Invest. 118, 3132-3142. 
Hacein-Bey-Abina, S., Hauer, J., Lim, A., et al. (2010). Efficacy of gene therapy for $\mathrm{X}$-linked severe combined immunodeficiency. N. Engl. J. Med. 363, 355-364.

Howe, S.J., Mansour, M.R., Schwarzwaelder, K., et al. (2008). Insertional mutagenesis combined with acquired somatic mutations causes leukemogenesis following gene therapy of SCID-X1 patients. J. Clin. Invest. 118, 3143-3150.

Hunter, M.J., Tuschong, L.M., Fowler, C.J., et al. (2011). Gene therapy of canine leukocyte adhesion deficiency using lentiviral vectors with human $\mathrm{CD} 11 \mathrm{~b}$ and $\mathrm{CD} 18$ promoters driving canine CD18 expression. Mol. Ther. 19, 113-121.

Kang, E.M., Choi, U., Theobald, N., et al. (2010). Retrovirus gene therapy for X-linked chronic granulomatous disease can achieve stable long-term correction of oxidase activity in peripheral blood neutrophils. Blood 115, 783-791.

Kang, H.J., Bartholomae, C.C., Paruzynski, A., et al. (2011). Retroviral gene therapy for X-linked chronic granulomatous disease: results from phase I/II trial. Mol. Ther. 19, 20922101.

Kerns, H.M., Ryu, B.Y., Stirling, B.V., et al. (2010). B cell-specific lentiviral gene therapy leads to sustained B-cell functional recovery in a murine model of $\mathrm{X}$-linked agammaglobulinemia. Blood 115, 2146-2155.

Lois, C., Hong, E.J., Pease, S., et al. (2002). Germline transmission and tissue-specific expression of transgenes delivered by lentiviral vectors. Science $295,868-872$.

Lombardo, A., Genovese, P., Beausejour, C.M., et al. (2007). Gene editing in human stem cells using zinc finger nucleases and integrase-defective lentiviral vector delivery. Nat. Biotechnol. 25, 1298-1306.

Malech, H.L., Maples, P.B., Whiting-Theobald, N., et al. (1997). Prolonged production of NADPH oxidase-corrected granulocytes after gene therapy of chronic granulomatous disease. Proc. Natl. Acad. Sci. U. S. A. 94, 12133-12138.

Marodon, G., Mouly, E., Blair, E.J., et al. (2003). Specific transgene expression in human and mouse CD4+ cells using lentiviral vectors with regulatory sequences from the CD4 gene. Blood 101, 3416-3423.

McCauslin, C.S., Wine, J., Cheng, L., et al. (2003). In vivo retroviral gene transfer by direct intrafemoral injection results in correction of the SCID phenotype in Jak3 knock-out animals. Blood 102, 843-848.

Montiel-Equihua, C.A., Zhang, L., Knight, S., et al. (2012). The beta-globin locus control region in combination with the EF1alpha short promoter allows enhanced lentiviral vectormediated erythroid gene expression with conserved multilineage activity. Mol. Ther. doi: 10.1038/mt.2012.50.

Moreau, T., Bardin, F., Imbert, J., et al. (2004). Restriction of transgene expression to the B-lymphoid progeny of human lentivirally transduced CD34+ cells. Mol. Ther. 10, 45-56.

Munoz, I.G., Prieto, J., Subramanian, S., et al. (2011). Molecular basis of engineered meganuclease targeting of the endogenous human RAG1 locus. Nucleic Acids Res. 39, 729-743.

Noguchi, M., Yi, H., Rosenblatt, H.M., et al. (1993). Interleukin-2 receptor gamma chain mutation results in $\mathrm{X}$-linked severe combined immunodeficiency in humans. Cell 73, 147-157.

Ott, M.G., Schmidt, M., Schwarzwaelder, K., et al. (2006). Correction of $\mathrm{X}$-linked chronic granulomatous disease by gene therapy, augmented by insertional activation of MDS1-EVI1, PRDM16 or SETBP1. Nat. Med. 12, 401-409.

Pai, S.Y., Notarangelo, L.D., Harris, C., et al. (2011). Somatic gene therapy for X-linked severe combined immunodeficiency using a self-inactivating modified gammaretroviral vector results in an improved preclinical safety profile and early clinical efficacy in a human patient. Blood 118: 164.

Pike-Overzet, K., Rodijk, M., Ng, Y.Y., et al. (2011). Correction of murine Rag1 deficiency by self-inactivating lentiviral vectormediated gene transfer. Leukemia 25, 1471-1483.

Sacco, M.G., Ungari, M., Cato, E.M., et al. (2000). Lymphoid abnormalities in CD40 ligand transgenic mice suggest the need for tight regulation in gene therapy approaches to hyper immunoglobulin M (IgM) syndrome. Cancer Gene Ther. 7, 1299-1306.

Santilli, G., Almarza, E., Brendel, C., et al. (2011). Biochemical correction of X-CGD by a novel chimeric promoter regulating high levels of transgene expression in myeloid cells. Mol. Ther. $19,122-132$.

Scaramuzza, S., Biasco, L., Ripamonti, A., et al. (2012). Preclinical safety and efficacy of human CD34(+) cells transduced with lentiviral vector for the treatment of Wiskott-Aldrich syndrome. Mol. Ther. doi: 10.1038/mt.2012.23.

Stein, S., Ott, M.G., Schultze-Strasser, S., et al. (2010). Genomic instability and myelodysplasia with monosomy 7 consequent to EVI1 activation after gene therapy for chronic granulomatous disease. Nat. Med. 16, 198-204.

Tahara, M., Pergolizzi, R.G., Kobayashi, H., et al. (2004). Transsplicing repair of CD40 ligand deficiency results in naturally regulated correction of a mouse model of hyper-IgM X-linked immunodeficiency. Nat. Med. 10, 835-841.

Thrasher, A.J., Hacein-Bey-Abina, S., Gaspar, H.B., et al. (2005). Failure of SCID-X1 gene therapy in older patients. Blood 105, $4255-4257$.

Throm, R.E., Ouma, A.A., Zhou, S., et al. (2009). Efficient construction of producer cell lines for a SIN lentiviral vector for SCID-X1 gene therapy by concatemeric array transfection. Blood 113, 5104-5110.

Van Til, N.P., De Boer, H., Mashamba, N., et al. Correction of murine Rag2 severe combined immunodeficiency by lentiviral gene therapy using a codon-optimized RAG2 therapeutic transgene. Mol. Ther. doi: 10.1038/mt.2012.110.

Yates, F., Malassis-Seris, M., Stockholm, D., et al. (2002). Gene therapy of RAG-2-/- mice: sustained correction of the immunodeficiency. Blood 100, 3942-3949.

Yu, P.W., Tabuchi, R.S., Kato, R.M., et al. (2004). Sustained correction of B-cell development and function in a murine model of X-linked agammaglobulinemia (XLA) using retroviralmediated gene transfer. Blood 104, 1281-1290.

Zhang, F., Frost, A.R., Blundell, M.P., et al. (2010). A ubiquitous chromatin opening element (UCOE) confers resistance to DNA methylation-mediated silencing of lentiviral vectors. Mol. Ther. 18, 1640-1649.

\author{
Address correspondence to: \\ Dr. Adrian J. Thrasher \\ Centre for Immunodeficiency \\ Molecular Immunology Unit \\ Institute of Child Health \\ 30 Guilford Street \\ London WC1N 1EH \\ United Kingdom
}

E-mail: a.thrasher@ucl.ac.uk

Received for publication June 9, 2012; accepted after revision June 11, 2012.

Published online: June 11, 2012. 